\title{
Difficulties in diagnosis and treatment of Paget's disease
}

\author{
ALEKSANDRA KAWALEC', в, D-F, ALEKSANDRA ZDROJOWY-WEŁNA², в, E, G, \\ GRAŻYNA BEDNAREK-TUPIKOWSKA², A, E \\ ${ }^{1}$ Salmed Specialized Clinic in Swiebodzice \\ ${ }^{2}$ Clinic of Endocrinology, Diabetology and Isotope Therapy, Medical University of Wroclaw, \\ Independent Public Teaching Hospital No. 1 in Wroclaw
}

A - Study Design, B - Data Collection, C - Statistical Analysis, D - Data Interpretation, E - Manuscript Preparation $\mathbf{F}$ - Literature Search, $\mathbf{G}$ - Funds Collection

Summary Paget's disease is a rare finding in Poland. It is a disorder of the osteoarticular system, which, in adults, mostly affects people over 55 years of age. The clinical picture varies, depending on the location of the lesions, making the diagnosis difficult, sometimes taking many years for a correct diagnosis to be made. In etiopathogenesis, genetic predispositions as well as viral infections play an important role. From the genetic point of view, Paget's disease is heterogeneous, as numerous mutations are known, and the genotype to phenotype relationship is unclear. The first phase of the disease is characterized by an increased osteolytic activity, due to morphologically changed and RANKL overstimulated osteocytes. This leads to an intensification of ossification processes that occur in a chaotic manner. Therefore, the resulting bone is weak, extensively vascularized and there is an increased risk of fracture or deformity. Clinical manifestations of Paget's disease might include pain, excessive warmth, bone deformations, degenerative lesions in the adjacent joints, compression of the neural structures, hearing loss, and dilated cardiomiopathy. Other possible complications include the development of benign and malignant bone tumors and hypercalcaemia in the case of immobilization. An elevated level of serum alkaline phosphatase, bone x-ray and bone scintigraphy are crucial in making the diagnosis. The disease should be distinguished from osteomalacia, osteoporosis, hyperparathyroidism and multiple myeloma. Bisphosphonates at doses higher than those applied for osteoporosis are an effective treatment. The occurrence of orthopedic, neurological and laryngological complications is often a reason for surgical intervention. Key words: Paget's disease, alkaline phosphatase, bisphosphonates.

Kawalec A, Zdrojowy-Wełna A, Bednarek-Tupikowska G. Difficulties in diagnosis and treatment of Paget's disease. Fam Med Prim Care Rev 2016; 18(4): 501-505, doi: 10.5114/fmpcr.2016.63712.

\section{Background}

Ailments of the osteoarticular system are one of the most common reasons for presentation to a general practitioner of mature and especially elderly people. Their causes are usually degenerative lesions, which are treated with nonsteroidal anti-inflammatory drugs and rehabilitation methods. Only a long-term observation and especially a radiological image of bones can reveal that the cause of the ailment may also be Paget's disease. The second group of patients who are diagnosed with this disease includes people with incidentally detected high levels of alkaline phosphatase. Such patients are often examined for bile duct diseases, in particular for cancer even if their general condition is good. Only comprehensive biochemical diagnostics and imaging studies of bones allow for a diagnosis of Paget's disease. Patients are usually first diagnosed by general practitioners, and subsequently by orthopedists, neurologists or rheumatologists, laryngologists and sometimes endocrinologists. Therefore, it is important for the physicians of the above-mentioned specialties to be especially mindful of the existence of this disease. This disease was first described by Sir James Paget in 1879.

\section{Definition}

Paget's disease (Osteitis deformans) is a disease of a local, mono- or multifocal nature, which in its initial phase is associated with activation of resorption and, subsequently, with ossification processes leading to disorganization of the bone structure and its deformation. The course of the disease can be asymptomatic, or it can involve ailments such as pain, fractures, degenerative lesions in joints, visible deformities, hearing loss and symptoms of compression of the neural structures. The disease affects mature people, often over 55 years of age. The etiology is not fully understood; there is a genetic predisposition, and viral infection is considered.

\section{Epidemiology}

Paget's disease often occurs in northern England, Western Europe, the United States, Canada, Australia and New Zealand. It is rarely found in Asia, Africa and Scandinavia. During the last 30 years, the incidence and severity of cases in the United Kingdom and New Zealand decreased for unclear reasons, possibly due to the migration of people from Asia. In countries of high incidence, it is the second most common metabolic bone disease after osteoporosis. It occurs in $1-2 \%$ of Caucasians over 55 years of age, and after 80 years of age, is present in as much as $5 \%$ of women and $8 \%$ of men [1].

\section{Etiopathogenesis}

The essence of Paget's disease is the intensification of the processes of osteolysis and, secondarily, of ossification. 
Osteoclastic cells - osteoclasts are formed from mononuclear precursors of the monocyte/macrophage line, which undergo a transformation to form multinuclear osteoclasts and are then activated for the bone resorption process. Proper reconstruction - bone remodeling is under the control of the RANKL/RANK/OPG (osteoprotegerin) system. This system regulates the formation and functions of osteoclasts. RANK is a receptor present on precursors of osteoclasts. RANKL is a cytokine present on osteoblasts and, when combined with RANK on the surface of osteoblast precursors, it results in the activation of secondary intracellular messengers: the nuclear factor $\mathrm{kB}(\mathrm{NK}-\kappa \mathrm{B})$, a system of protein kinases and ERK. Each of these activation pathways is associated with the differentiation, function and survival of osteoclasts. OPG is a trap receptor, which binds RANKL and prevents the combination of RANKL with RANK. Thus, OPG inhibits osteoclast differentiation. Factors affecting the expression of RANKL include: active vitamin $\mathrm{D}_{3}-1,25-(\mathrm{OH})_{2} \mathrm{D}_{3}$, interleukin 1 and 11, parathyroid hormone.

Paget's disease is a disease of increased bone resorption, and osteoclasts exhibit morphological changes and dysfunctions. In the affected areas of the bone, they have an increased number and size of nuclei - over 100, while normal osteoclasts contain 3-20 nuclei. Another feature of the nuclei of osteoclasts includes intranuclear inclusions resembling paramyxovirus nucleocapsids. Osteoclasts in patients with this disease over-respond to RANKL. In the areas affected by the disease, the number of osteoblasts which are morphologically and functionally normal increases.

In response to the resorption, compensatory ossification takes place, leading to increased bone turnover. New collagen fibers are not arranged linearly, but in a disorganized manner, resulting in an impaired bone architecture, which is wooly and lamellar, and is often thickened, with an increased number of blood vessels. Disorganization of the bone structure results in its mechanical weakness and increased incidence of fractures and deformities [2].

\section{Genetics}

Specific geographical and familial occurrence of the disease suggests its genetic background and the role of environmental factors in the etiology of the disease [3]. Cases of sporadic occurrence of this disease are also known. In families affected by the disease, mutations in different locations in the genome were demonstrated - the disease is genetically heterogeneous. Mutations concern genes encoding RANK/NF- $\kappa B$ - the pathway activating osteoclasts [2].

The pattern of heredity is autosomal dominant. In first-degree relatives of a patient suffering from Paget's disease, the risk of the disease is seven times higher compared to individuals without family-related risk factors. It was demonstrated that clinical signs of the disease can be determined in $15 \%$ of family members, but radiological and scintigraphic signs can be determined in up to $40 \%$ of first-degree relatives [3].

It has been found that the disease is associated with mutations of the following locations in the genome:

- chromosome 6p [4],

- chromosome 18q22.1 - TNFRSF11A gene encoding RANK,

- chromosome 5q35 - SQSTMI gene,

- chromosome $5 \mathrm{q} 31$.

The SQSTMI gene mutation was identified in Canada, in families originated from France. It is present in $50 \%$ of patients with a familial form of the disease and in $16 \%$ of patients with its sporadic form. In turn, in the United Kingdom, this mutation has a prevalence of $19 \%$ in the familial form and $9 \%$ in the sporadic form. The product of the gene is p62 protein, activating osteoclasts by a nuclear factor kappa B.
However, not all people with the SQSTMI gene mutation will suffer from Paget's disease [3, 5].

The TNFRF11B gene mutation, causing a deficiency in OPG in its homozygous form, is responsible for the juvenile form of Paget's disease. This is the only example of a close genotype-to-phenotype relationship in Paget's disease [6].

The development of the disease is most probably strongly affected by viral infection [2]. In osteoclasts in Paget's disease, microfilaments identical to paramyxovirus nucleocapsids, in particular of the measles virus, were demonstrated. This gave rise to the idea that the disease may be related to a latent viral infection. However, osteoclasts are not self-renewing cells, so other types of cells would have to be a reservoir of the measles virus from childhood to an elderly age [2].

The accumulation of cases of Paget's disease in Lancashire in England and in Australia suggests that, most likely, environmental factors are also important [4].

\section{Clinical symptoms}

The description of Paget's disease concerned a patient whose disease began at the age of 46 with pain in the lower extremities, and after two years, a nodular bone thickening appeared on the left tibia, and subsequently a similar one was observed within the left femur. The lesions were periodically painful; the pain was not associated with compression. After three years, the patient still enjoyed good health, but his left tibia underwent thickening and visible deviation. Similar changes occurred in the femur, and the patient could not join both knees. The lesions also concerned the pelvis, which was widened and flattened. As a result, the lower extremity shortened. The patient noticed an enlargement of the skull on the right side; his hats were becoming too tight. Despite the progressive changes, the patient did not suffer; he was walking, riding a horse and was involved in the daily activities of a gentleman living in the countryside. For the following 17 years, the disease progressed slowly; the left femur became thicker, heavier, more curved, and the right leg underwent similar deformations. The mobility of the knee joints decreased, and the skull underwent further enlargement, but it preserved its natural shape. The spine strongly deviated backward in its upper section and forward in its lower section, and height decreased. The chest constricted laterally, and the respiratory movement of the ribs decreased. The figure underwent particular changes: his head bent so that it extended below the upper edge of the sternum. In this period, mitral insufficiency was diagnosed, the patient suffered from exudative inflammation of the left knee, a hemorrhage to the retina of both eyes, gradual hearing loss, and he complained of neuralgic pains. In the last period of life, he was diagnosed with a malignant tumor of the left forearm bone. Despite progressive bone lesions, the patient defined his general health condition as "excellent". The patient died as a result of effusion into the right pleural cavity. During the autopsy, the malignant tumor of the left radial bone was confirmed, as well as metastatic lesions to the right pleura and a significantly enlarged heart of thinned walls, and a significant thickening of the bones of the skull was found. In the bones affected by the disease, an increased number of vessels was demonstrated; their structure was hard and porous [7]. The classic clinical picture of the disease presented by Sir J. Paget is rare nowadays [1].

Currently, the disease is most often asymptomatic and is diagnosed through an incidental finding of radiological lesions in bones or an elevated level of alkaline phosphate. Most often, the axial skeleton is affected - especially the lumbosacral spine, pelvis, skull, as well as the femur and tibia [3]. A common symptom is pain in the affected extremity, increasing due to microfractures. The pain is not related 
to physical activity, and it intensifies at night. In the course of the disease, the development of bone deformity with excessive warming of the skin over the affected bone due to increased vascularization occurs. A complication of bone deformity may be a fracture. A common manifestation is an arched curvature of the femurs and lower legs loaded by body weight because of gravity.

If bone lesions affect the area of the joints, the development of degenerative and distortive lesions, especially of knee and hip joints, with a significant reduction in their mobility, is often present.

The involvement of the skull, including its thickening, results in an enlargement of the circumference of the head, pain, difficulty in maintaining the head in a vertical position, hearing loss, symptoms of compression of the cranial nerves, symptoms of the compression of the posterior cranial fossa structures in the form of ataxia, muscle weakness and respiratory disorders.

In the case of the involvement of the spine, the bodies and their processes enlarge, then compression fractures occur, and symptoms of compression of the spinal cord and spinal nerve roots may appear.

Another complication of the disease is dilated cardiomyopathy, which may be associated with bone remodeling and increased vascularization [3].

Within bone lesions, there is an increased risk of the development of sarcomas, which is estimated at $1 \%$, although this can be increased to $10 \%$ in a subpopulation with extensive involvement of the skeleton. A suspicion of a sarcoid lesion results from the appearance of pain and edema of the affected bone. It is most often an osteosarcoma, fibrosarcoma or anaplastic sarcoma. The prognosis is bad, because the 5 -year survival rate is $7.5-10 \%$.

Other tumors developing more frequently in the course of Paget's disease are giant-cell tumors of the bone. They are less common than sarcomas, are benign and are treatable with surgery or radiotherapy.

In patients immobilized for a lengthy period of time due to of fractures, hypercalcaemia is a common complication [5]. The reason for this is increased bone resorption as compared to the impaired ossification in situations of no mechanical stimulation [8].

\section{Histological picture}

The primary disorder is increased focal bone resorption. In resorption bays (Howship's lacunae), an increased number of enlarged osteoclasts with an increased number of nuclei is present. The volume of the cortical and cancellous bone is reduced, the course of osteons becomes tortuous, and the bone marrow within the lesions is displaced by a richly vascularized fibrous tissue. Reactively stimulated ossification, with an increase in the number of osteoblasts, results in the formation of characteristically arranged bone fragments, leading to an irregular mix of woolly and lamellar bone. In the final period of the disease, inhibition of remodeling takes place, leaving a chaotic pattern of the osteoid matrix, practically without any cellular activity [5].

\section{Diagnostics}

From a clinical point of view, the best marker of the disease is an elevated level of the bone fraction of alkaline phosphatase, an enzyme produced by osteoblasts. Its activity correlates with the extent of the lesions and can be used to monitor the effects of treatment [5]. It is an inexpensive and easily available examination. As high concentration of alkaline phosphatase may be associated with liver diseases, it is necessary to determine bone isoenzyme. In patients with minor lesions, its concentration may be normal (approx. $10 \%$ of cases), which does not exclude the disease [3]. In such a case, in order diagnose and monitor the course of the disease, it is useful to keep track of the concentration of bone isoenzyme of alkaline phosphatase, which is usually increased - higher concentrations are observed in $60 \%$ of patients whose disease is limited to just one bone [9]. Other markers of increased bone turnover include: specific markers of collagen I degradation, N-telopeptide and C-telopeptide, hydroxyproline, pyridinoline and deoxypyridinoline. Considering the fact that approximately $70 \%$ of patients do not have clinical symptoms, in practice, patients with a very high level of alkaline phosphatase who haven't been diagnosed for many years are occasionally encountered. This results from the rare occurrence of Paget's disease in Poland, along with the resulting low vigilance of physicians in the search for this disease [5]. In a differential diagnosis, it is helpful to determine the concentration of calcium, phosphates and parathyroid hormone (Table 1).

In the diagnosis, radiological images of affected bones and scintigraphy are important. A radiological image allows for the assessment of bone lesions and helps in differentiation of the cause of bone pain (fractures, deformities, secondary degenerative changes). In the initial period, limited osteolytic foci dominate, which merge over time to form cystic areas. In the bones of the skull, these changes are well defined and described as osteoporosis circumscripta. In the long bones, the areas of osteolysis are well defined in the metaphyseal areas - comma-shaped cortical defects gradually spread in the direction of the bodies and produce larger formations in the shape of the letter $\mathrm{V}$. A secondary activation of ossification causes a thickening of the cortical layer, enlargement of external bone dimensions and axis disorders and deformities. In the bones of the skull, osteolytic and osteosclerotic foci are visible, which produce the saltand-pepper image. Over time, osteosclerotic changes begin dominating [5].

Scintigraphy with the use of $99 \mathrm{mTc}$-labeled bisphosphonates allows for the assessment of the extent and location of sites of the active disease, even in patients with normal levels of alkaline phosphatase [3]. Sites of increased uptake indicate increased bone turnover and increased vascularity. This examination is also particularly useful in assessing the effectiveness of treatment $[8,9]$.

Computed tomography and magnetic resonance imaging may be useful in case of symptoms of spinal stenosis, compression of the nerve roots, vertebral compression fractures and suspected malignant lesions $[3,8]$. In the latter

\begin{tabular}{|c|c|c|c|c|c|}
\hline & Calcium & Phosphorous & Alkaline phosphatase & PTH & X-ray/densitometry \\
\hline Osteoporosis & normal & normal & normal & normal & decreased BMD \\
\hline Osteomalacia & $\downarrow$ & $\downarrow$ & $\uparrow$ & $\uparrow$ & Looser-Milkmann zone \\
\hline Hyperparathyroidism & $\uparrow$ & $\downarrow$ & $\uparrow$ & $\uparrow$ & brown bone tumors \\
\hline Paget's disease & normal & normal & $\uparrow$ & normal & abnormal bone architecture \\
\hline Multiple myeloma & normal $/ \uparrow$ & normal $/ \uparrow$ & $\downarrow$ & normal & osteolytic foci \\
\hline
\end{tabular}


case, a PET examination with fluorodeoxyglucose may also be helpful [5].

Biopsy of the affected sites in bones is usually not necessary. The examination is only needed in the differential diagnosis of cancerous changes with a single sclerotic lesion in the vertebra, in the case of atypical locations and in the case of the appearance of a new lesion in the bones [3].

\section{Treatment}

In the treatment strategy of Paget's disease, a short-term procedure aimed at controlling the activity of the disease, reducing pain and neurological symptoms, and long-term therapy, aimed at inhibiting the progression of the disease, reducing the risk of deformity, fractures, degenerative lesions, pain and carcinogenesis, should be distinguished [5].

The treatment of choice includes bisphosphonates. This medication inhibits bone resorption performed by osteoclasts and is effective at reducing the metabolic activity of the disease [3]. Bisphosphonates have a cytotoxic effect on osteoclasts and cause a decrease in RANKL. The indication for the treatment is bone pain, progressive skeletal deformity, ailments caused by spinal stenosis, high activity of alkaline phosphatase, hypercalcaemia in the course of immobilization, prevention of long bones fractures and development of complication, especially when the vertebrae and skull are affected. In the case of the location of lesions in close vicinity to the joints, therapy is applied to prevent the development of degenerative lesions. Another indication is preparing for the operation, as this reduces the blood supply to the affected bone. This therapy is also applied in cases when the disease is diagnosed at a young age. This medication is also administered in the prevention of hearing impairment or loss if the disease affects the temporal bone. In the case of the occurrence of paralysis associated with Paget's disease affecting the spine, immediate intravenous therapy with bisphosphonates and consultation with a neurosurgeon for the need of surgery is recommended [9].

Doses of bisphosphonates in Paget's disease are higher than those used in osteoporosis [5]. Alternatively, risedronate is recommended orally, at a dose of $30 \mathrm{mg} / 24 \mathrm{~h}$ for 2 months, or alendronate, at a dose of $40 \mathrm{mg} / 24 \mathrm{~h}$ for 6 months. It is also possible to administer tiludronate orally, at a dose of $400 \mathrm{mg} / 24 \mathrm{~h}$ for 3 months, or pamidronate by intravenous infusion, at a dose of $30 \mathrm{mg}$ for $4 \mathrm{~h}$ for 3 consecutive days or at a dose of $60-90 \mathrm{mg}$ by a 24 -hour infusion for 2 consecutive days. Zolendronic acid is administered at a dose of $5 \mathrm{mg}$ by a single intravenous infusion over $15 \mathrm{~min}$ [5]. The side effects of bisphosphonates when used orally include nausea, abdominal pain, diarrhea or esophagitis. Products administered in an intravenous form may cause flu-like symptoms, bone pain, hypocalcaemia, atypical trochanteric femur fracture or necrosis of the jaw. These last two symptoms occur rarely in patients with Paget's disease due to the use of intermittent therapy. Other complications include kidney failure and atrial fibrillation. Bisphosphonates cannot be used when the creatinine clearance values are below $30 \mathrm{ml} / \mathrm{min}$ nor during pregnancy [3].
The efficacy of the treatment is assessed after 3 and 6 months after treatment with risedronate and alendronate, after 6 months of treatment with pamidronate and after one year of using zolendronic acid. If the symptoms return or the concentration of alkaline phosphatase increases by $75 \%$ above the upper limit of the normal level, the treatment should be repeated [8]. Bisphosphonates reduce bone turnover, pain intensity, decrease concentration of alkaline phosphatase and decrease radiological losses, but they do not prevent the progression of deformity and do not restore normal bone structure [3]. It was demonstrated that after a single dose of zolendronic acid, the therapeutic effect, in the form of normal bone turnover marker activity, persists for at least 2 years $[5,8]$. Data on the efficacy of bisphosphonate treatment is not consistent. In the PRISM study performed on 1,324 individuals in the United Kingdom, no difference in the number of fractures, orthopedic surgeries, quality of life or hearing loss was demonstrated within 2 to 5 years of observation in patients treated with bisphosphonates as compared to patients treated symptomatically. However, a significant difference in the concentration of alkaline phosphatase between the groups was demonstrated [10].

In the case of patients who cannot use bisphosphonates, a subcutaneous or intramuscular administration of calcitonin at a dose of 50-100 IU per day is recommended [3]. In patients with impaired renal function, Denosumab was used, which proved to be effective in reducing pain and decreased bone turnover [11].

\section{Surgical treatment}

In the case of advanced secondary degenerative lesions, especially in hip and knee joints, treatment with alloplasty is used. In the case of an arched deformity of femurs, it is possible to perform a corrective osteotomy. In the case of central cord syndrome or compression of the nerve roots, improvement can be made after neurosurgical procedures. After hearing loss, it is possible to perform surgery of the malleus immobilization, stapedectomy and cochlear implantation [5].

\section{Summary}

Paget's disease may have an asymptomatic course for many years, but it usually leads to irreversible bone deformities, fractures and neurological symptoms. Therefore, early diagnosis and treatment are important. In Poland, this disease is rare, but there may be much more cases. The difficulty in diagnosis results from the diversity of clinical symptoms associated with different locations of bone lesions [12]. Another problem is diagnosis in an asymptomatic patient with a high level of alkaline phosphatase and without perceptible liver diseases. Paget's disease should be considered in the differential diagnosis in patients in good general condition over 55 years of age. Usually, basic tests of bone metabolism, X-ray of the affected bones and scintigraphy are sufficient for making a diagnosis.

Source of funding: This work was funded by the authors' resources. Conflict of interest: The authors declare no conflict of interests.

\section{References}

1. Britton C, Walsh J. Paget disease of bone. Aust Fam Physician 2012; 41(3): 100-103.

2. Sabharval R, Gupta S, Sepolia S, et al. An insight in to Paget's disease of bone. Niger J Surg 2014; 20(1): 9-15.

3. Seton M. Paget disease of bone: diagnosis and drug therapy. Cleve Clin J Med 2013; 80(7): 452-462. 
4. McKuscik V, Hamosh A. Paget disease of bone PDB. Cytogenetic locations: 5q35, 5q31, $18 q 22$ [cited 08.07.2015]. Avaible from URL: http://www.omim.org/entry/602080.

5. Misiorowski W. Choroba Pageta kości. W: Zgliczyński W, ed. Wielka Interna. Endokrynologia. T. 11, Cz. 1. Warszawa: Medical Tribune Polska; 2011: 436-439.

6. McKusick V, O'Neill MJF. Paget disease, juvenile. Cytogenetic locations: 8q24 [cited 08.07.2015]. Avaible from URL: http:// www.omim.org/entry/239000.

7. Paget J. On a form of chronic inflammation of bones (osteitis deformans). Med Chir Trans 1877; 60: 37-64.9.

8. Ferraz-de-Souza B, Correa PH. Diagnosis and treatment of Paget's disease of bone: a mini review. Arq Bras Endocrinol Metabol 2013; 57(8): 577-582.

9. Singer FR, Bone HG, Hosking DJ, et al. Paget's disease of bone: an endocrine society clinical practice guideline. J Clin Endocrinol Metab 2014; 99(12): 4408-4422.

10. Langson AL, Cambell MK, Faser WD, et al. Randomized trial of intensive bisposphonate treatment versus symptomatic management in Paget's disease of bone. J Bone Miner Res 2010; 25(1): 20-31.

11. Schwarz P, Rasmussen AQ, Kvist TM, et al. Paget's disease of the bone after treatment with Denosumab: a case report. Bone 2012; 50(5): 1023-1025.

12. Almeida LP, Scrignoli JA, Santos SC, et al. Paget's disease of bone and its complications due to delay in diagnosis. Acta Reumatol Port 2011; 36(3): 288-291.

Tables: 1

Figures: 0

References: 12

Received: 10.07.2015

Revised: 14.07.2015

Accepted: 14.07.2015

Address for correspondence:

Aleksandra Kawalec, MD

Salmed Przychodnia Specjalistyczna

Aleksandra Kawalec

ul. Królowej Elżbiety 84

58-160 Świebodzice

Polska

Tel.: +48 600 341-649

E-mail: a.kawalec70@gmail.com 